\title{
Soliton content in the standard optical OFDM signal
}

\author{
Egor V. Sedov ${ }^{1}$, Alexey A. Redyuk ${ }^{1,2}$, Mikhail P. Fedoruk ${ }^{1,2}$, Andrey A. Gelash ${ }^{1,3}$, Leonid L. \\ FRUMIN $^{1,4}$, AND SERGEY K. TURITSYN ${ }^{1,5, *}$ \\ ${ }^{1}$ Novosibirsk State University, Novosibirsk, 630090, Russia \\ ${ }^{2}$ Institute of Computational Technologies, Siberian Branch, Russian Academy of Sciences, Novosibirsk, 630090, Russia \\ ${ }^{3}$ Kutateladze Institute of Thermophysics, Siberian Branch, Russian Academy of Sciences, Novosibirsk, 630090, Russia \\ ${ }^{4}$ Institute of Automation and Electrometry, Siberian Branch, Russian Academy of Sciences, Novosibirsk, 630090, Russia \\ ${ }^{5}$ Aston Institute of Photonic Technologies, Aston University, Birmingham, B4 7ET, UK \\ ${ }^{*}$ Corresponding author: s.k.turitsyn@aston.ac.uk
}

Compiled November 10, 2018

Nonlinear Schrödinger equation (NLSE) is often used as a master path-average model for fiber-optic transmission lines. In general, NLSE describes the co-existence of dispersive waves and soliton pulses. Propagation of signal in such a nonlinear channel is conceptually different from linear systems. We demonstrate here that the conventional orthogonal frequency-division multiplexing (OFDM) input optical signal at powers typical for modern communication systems might have soliton components statistically created by the random process corresponding to the information content. Applying Zakharov-Shabat spectral problem to a single OFDM symbol with multiple sub-carriers we quantify the effect of statistical soliton occurrence in such an information-bearing optical signal. Moreover, we observe that at signal powers optimal for transmission OFDM symbol incorporates multiple solitons with high probability. The considered optical communication example is relevant to a more general physical problem of generation of coherent structures from noise. () 2018 Optical Society of America

OCIS codes: (190.5530) Pulse propagation and temporal solitons; 060.2330 Fiber optics communications; (070.4340) Nonlinear optical signal processing;

http://dx.doi.org/10.1364/ao.XX.XXXXXX

\section{INTRODUCTION}

Optical fiber is a remarkable engineered physical medium important for a range of practical applications, including telecommunications, sensing, lasers, imaging and many others [1]. Light trapped in silica waveguide can propagate with extremely low field attenuation over long distances. Optical fiber medium can also act as a nonlinear system when signal accumulates during the propagation a noticeable (of the order of $\pi$ ) nonlinear phase change due to the fiber Kerr effect. In some applications, such as mode-locked fiber lasers, the nonlinear Kerr effect is used positively, providing conditions for mode-locking and pulse shaping in laser. In modern telecommunication systems, nonlinearity is typically considered as the factor limiting their performance at high signal-to-noise ratio.

The nonlinear properties of the fiber communication links create a number of unusual (compared to linear channels) challenges. However, channel nonlinearity also offers new interesting opportunities. It is well known, that the nonlinear Schrödinger equation (NLSE) describes under particular conditions and within certain limits the propagation of a signal down an optical fiber [1-5]. Written in the generic normalized form (see for detail e.g. [1-3]) the NLS equation reads

$$
i \frac{\partial q}{\partial z}+\frac{1}{2} \frac{\partial^{2} q}{\partial t^{2}}+|q|^{2} q=0
$$

In the context of fiber-optic, we consider here the case of the so-called anomalous dispersion, when general solutions of this equation can include both the dispersive (linear-like) waves and the coherent structures - solitons. Any initial field evolving according to this master model can be presented as a nonlinear superposition of dispersive waves and soliton(s).

In 1972, Zakharov and Shabat demonstrated that the NLSE can be integrated by the inverse scattering transform (IST) method [6], also known nowadays as the nonlinear Fourier transform (NFT). The IST/NFT allows one to present field (with the evolution along distance $z$ governed by NLSE) at an arbitrary distance using nonlinear spectrum of the initial (at $z=0$ ) signal distribution. More specifically, nonlinear spectrum of the initial field $q(t, z=0)$ can be found through the solution of the Zakharov-Shabat spectral problem:

$$
\left\{\begin{aligned}
-\partial_{t} \psi_{1}+q(t, 0) \psi_{2} & =i \xi \psi_{1} \\
\partial_{t} \psi_{2}+q^{*}(t, 0) \psi_{1} & =i \xi \psi_{2}
\end{aligned}\right.
$$

$q(t, 0)=q_{0}(t)$ is the "potential" - initial distribution of the signal, $\psi_{1,2}$ is a vector eigenfunction and $\xi$ - spectral parameter defined on a complex plane.

In general, the nonlinear spectrum for the localized in time domain optical signal $q_{0}(t)$ has discrete eigenvalues and continuous component corresponding to the spectrum of the system (2). The continuous spectrum of the system (2) fills the real axis of the $\xi$-plane and corresponds to the dispersive wave component, which will not be our focus here. 
The discrete spectrum eigenvalues $\xi_{n}$ correspond to soliton solutions of the NLSE. For some classes of initial initial pulse, there are known analytical and numerical results concerning the soliton content in the initial field $q_{0}(t)$, see for detail e.g. [7-9]. In the case of a real-valued unmodulated (no temporal dependence of the phase) rectangular pulse, the number of solitons $N$ containing in such field can be found as

$$
N=\operatorname{int}\left[1 / 2+L_{1}(q) / \pi\right]
$$

Where $\operatorname{int}[\ldots]$ means the integer part of the expression and $L_{1}(q)=\int_{-\infty}^{+\infty}|q(t)| d t$ is the (non-dimensional) signal $L_{1}$ norm. Evidently, the $L_{2}$ norm given by $L_{2}=\int_{-\infty}^{+\infty}|q(t)|^{2} d t$ corresponds to the signal energy. In the case of more complex initial signals the analytical approaches are limited and analysis requires extensive statistical numerical modelling based on direct solving of Zakharov-Shabat spectral problem (2). For instance, in [10] generation of solitons from noise and noncoherent optical pulses has been considered using $L_{2}$ norm as a measure. Analysis of a soliton content in chirped Gaussian pulses was done in [11] and in the optical speckle fields in [12]. In particular, it was shown that modulation of a simple rectangular pulse leads to significant decrease of the number of emerging solitons [10].

It is well known that an information-bearing signal can be treated as a random process, in which signal characteristics, such as power and phase, experience statistical variations that depend on modulation formats and coding [13]. Here we study soliton content in a standard optical orthogonal frequency-division multiplexing (OFDM) signal, in which digital data is encoded on multiple carrier frequencies. We are interested here only in the total number of the discrete eigenvalues, but not in their specific parameters. Therefore, we apply the method described in [14], which links the number of discrete eigenvalues to the total phase shift of the coefficient $a(\xi)$ by the formula:

$$
N=\left.\frac{1}{2 \pi} \operatorname{Arg}(a(\xi))\right|_{-\infty} ^{+\infty}
$$

the spectral parameter $\xi$ takes values from $-\infty$ to $+\infty$ on the real axis. The coefficient $a(\xi)$ is one of the coefficients characterizing the scattering on the "potential" $q(t, 0)$ in the Zakharov-Shabat problem (2), see for detail e.g. [15] and also recent work [16].

We would like to stress that in the framework of the considered integrable NLS equation model, there is no need for numerical simulations of initial signal propagation with distance $z$. The number of discrete eigenvalues will not be changed during propagation governed by the NLS equation. Moreover, parameters of continuous and discrete nonlinear spectrum will be changed in a trivial manner [5]. Therefore, we focus in this Letter on the analysis on the solutions of the Zakharov-Shabat spectral problem, rather than on consideration of the propagation dynamics of the field.

\section{SOLITON CONTENT IN THE CONVENTIONAL OFDM OPTICAL SIGNAL}

Orthogonal frequency-division multiplexing combines multiplexing and modulation. A single OFDM symbol (over time interval with duration $\mathrm{T}$ ) is presented as a sum of independent sub-carriers:

$$
s(t)=\sum_{k=0}^{M-1} X_{k} e^{i 2 \pi k t / T}, 0 \leq t<T,
$$

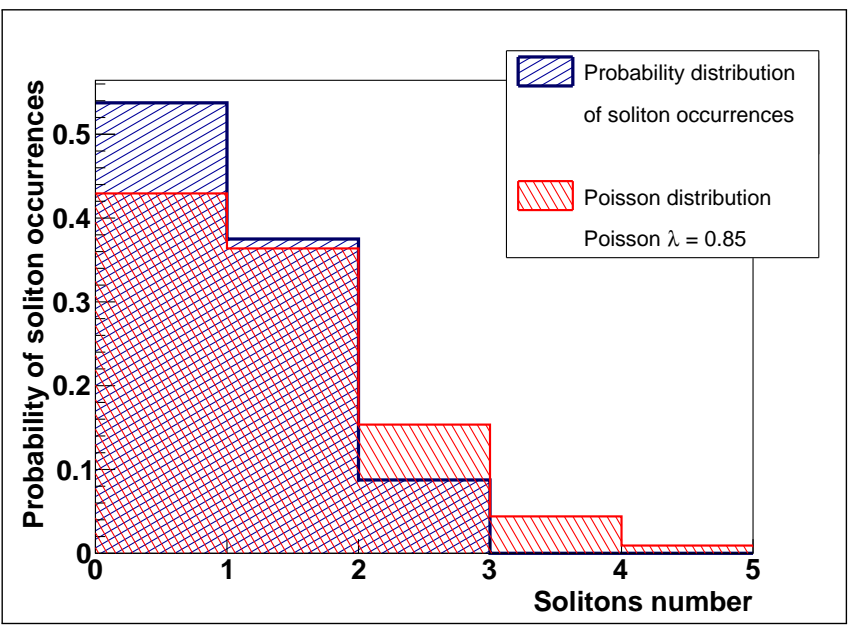

Fig. 1. Probability distribution of soliton occurrences in OFDM symbol with QPSK modulation, 128 sub-carriers and with average power of $-18 \mathrm{dBm}$

here $X_{k}$ corresponds to digital data, $\mathrm{M}$ is the number of subcarriers and $\mathrm{T}$ is the symbol interval. In practice, the number of subcarriers is selected as $M=2^{p}$ to use the Fast Fourier Transform (FFT) algorithm. In the real world units, we examine OFDM symbol with $10 \mathrm{~ns}$ symbol duration and QPSK or 16QAM modulation. Full FFT size is 1024 and number of sub-carriers $\mathrm{M}$ is changing from 16 to 1024 . Average signal power is linked to the $L_{2}$ norm (in dimension units) as following: $P_{\text {ave }}=L_{2} / T$, and it varies (in the dimension units) from -21 to $-8 \mathrm{dBm}$.

Without loss of generality, we focus here on two types of popular modulation formats: quadrature amplitude modulation (QAM) and phase-shift keying (PSK) and consider a single OFDM symbol (i.e. assuming burst mode transmission with well separated symbols). We analyze the probability of the appearance of solitons in the input OFDM symbol, depending on the signal parameters: modulation type, $L_{1}$ or $L_{2}$ norms and number of sub-carriers $M$. We use in the numerical simulations shown in Fig. 5 the following typical parameters: group velocity dispersion parameter $\beta_{2}=-21.5$ (in $\mathrm{ps}^{2} / \mathrm{km}$ ) and the nonlinear Kerr coefficient $\gamma=1.27$ (in $W^{-1} \mathrm{~km}^{-1}$ ). We accumulate statistics on the number of occurred solitons for fixed system and signal parameters by varying input digital data. Each graph point corresponds to 160 statistical measurements. For example, Fig. 1 shows the probability distributions for OFDM signal with QPSK modulation at 128 subcarriers with $P_{\text {ave }}=-18 \mathrm{dBm}$, and also Poisson fit distribution $\left(P(x ; \lambda)=e^{-\lambda} \cdot \lambda^{N} / N\right.$ !) for this data ( $\lambda$ is extracted from the data fitting), obtained by numerical simulation (the number of events equals $1,000,000$ ).

We examine now the probability of occurrence of solitons in the OFDM signal defined as the ratio of the number of symbols containing discrete eigenvalues (corresponding to solitons) of the Zakharov-Shabat spectral problem to the total number of the considered OFDM symbols. In other words, we are not interested in the exact number of solitons in the signal, but rather in their existence in the given OFDM symbol. Our goal here is to demonstrate that appearance of solitons in the OFDM signal is not something exotic, but rather is a general situation at certain practical power levels. We did verify that number of solitons does not depend on increase of the computational grid and the FFT size (temporal signal discretization).

Figure 2 shows the probability of the occurrence of solitons at 


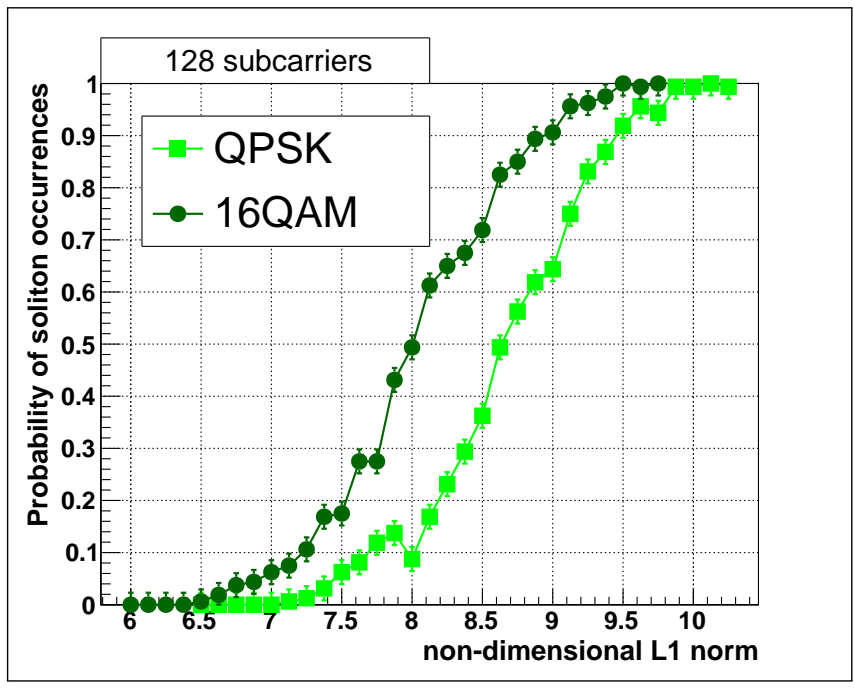

Fig. 2. Average number of occurred solitons versus value of $L_{1}$ norm for OFDM signals with QPSK and 16QAM modulations. The first threshold of $L_{1}$ norm value, calculated using the formula (3), is 1.57 and lies on the left well outside the boundaries of the graph

128 subcarriers versus the $L_{1}$ norm value. Note that the probability of the soliton appearance for signals with the same $L_{1}$ norm value is higher for signals with 16QAM modulation compared to QPSK. This trend is maintained for all numerical modeling with various parameters. It is also seen that for such highly modulated complex signals threshold of soliton occurrence is much higher than the analytical results (3) obtained for real unmodulated rectangular smooth functions.

Figures 3 and 4 show how the probability of occurrence of the soliton content in the OFDM signal is growing with the increase of the average power $P_{a v e}$. One can see that in signals with 128 subcarriers, solitons start to emerge at lower values of the norm compared to signals with 1024 subcarriers. It is seen that this effect depends on the number of subcarriers and signal modulation format. The transition from random (depending on the information content) appearance of solitons in certain (relatively rare) OFDM signals to the regime where most of the symbols contain discrete eigenvalues (soliton component) does happen over the interval of $3-4 \mathrm{dBm}$ increase of input signal power. An interesting observation is that it does not require too high signal power, for soliton component to become an inherent part of the OFDM symbol. The soliton component arises at rather practical levels of a signal power conventional for telecom applications.

We would like to stress that solitons appear in the OFDM signal not at very high powers. As a matter of fact, a soliton content is present in the OFDM signals at the powers optimal for transmission. To illustrate this point, we considered a $1000 \mathrm{~km}$ and $2000 \mathrm{~km}$ transmission links based on ideal distributed Raman amplification scheme with continuous amplified spontaneous emission generation (see [4,5] for detail). As an input, we used 16QAM-OFDM signal with 128 subcarriers and $10 \mathrm{~ns}$ symbol duration. At the receiver, the chromatic dispersion was fully compensated and an algorithm based on the 4th-power ViterbiViterbi method was used for phase estimation. System performance was evaluated using the parameter $Q^{2}$-factor, which measures the quality of an transmission signal. The $\mathrm{Q}^{2}$-factor

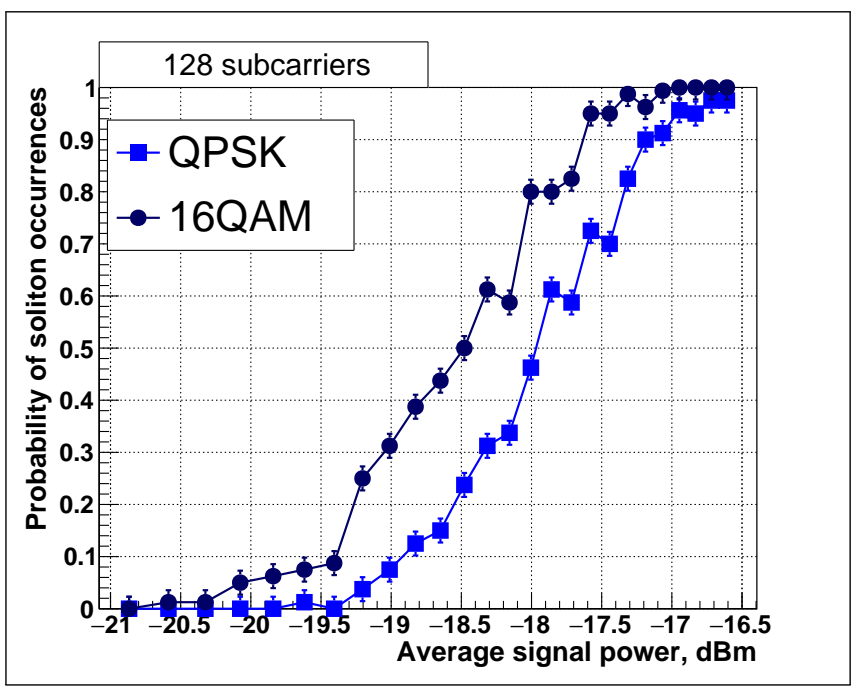

Fig. 3. Average number of solitons embedded into OFDM symbol with 128 subcarriers and QPSK and 16QAM modulations versus average signal power.

value has been extrapolated from the conventional error vector magnitude (EVM) function [17] as $Q^{2}=1 / E V M^{2}$ using transmission of $2^{14}$ OFDM symbols per run. Results are shown in Fig. 5. Optimal transmission is achieved with an average power around $-15 \mathrm{dBm}$, that according to Fig. 3 is well in the regime where a soliton component in the OFDM symbol is highly likely.

In general, solitons and dispersive waves propagate in a different manner down the optical fiber. The most noticeable difference is that in the soliton, dispersive broadening is counterbalanced by the nonlinear effects. Therefore, the presence of solitons embedded into the conventional OFDM symbol potentially should impact the transmission of the combined signal. Comprehension of this fact and its consequences for signal coding, modulation and processing might be important for the improvement of the performance of fibre-optic communication systems. However, it should be pointed out that effect of the embedded solitons does not lead to a drastic change of the symbol propagation dynamics. Further study is required to understand how the presence of solitons affects transmission and how it can be used in practical terms. Note that even for quasi-linear signal propagation statistical fluctuations in the plane $(z, t)$ might be very nontrivial and are dependent on the information data, format and modulation, see e.g. recent publication [18].

\section{CONCLUSIONS}

Traditional signal modulation formats have been designed and developed for linear communication channels. Transmission in the nonlinear channel reveals rather unusual properties of such conventional signals. Considering the NLSE as a master channel model, we have shown here that a standard OFDM signal statistically contains soliton components at powers of practical interest. Using Zakharov-Shabat spectral problem, we studied statistics of soliton occurrences in OFDM signal and quantify how the number of solitons that are embedded into the input OFDM signal increases with the $L_{1}$ norm and signal average power.

This observation indicates that transmission in a nonlinear channel substantially changes the whole paradigm of signal modulation and processing. Our results show that nonlinear 


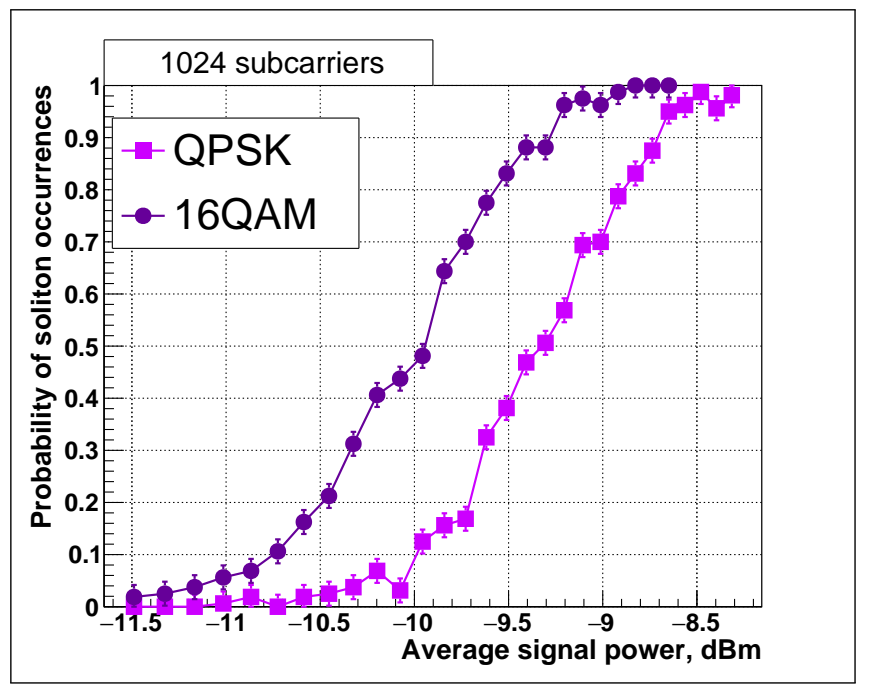

Fig. 4. Average number of solitons embedded into OFDM symbol with 1024 subcarriers and QPSK and 16QAM modulations versus average signal power.

analysis might be useful not only to special inherently solitonbased systems and signals [3], but also for conventional communication formats that traditionally are not linked to the soliton concept and techniques. We believe that our results indicate that application of the detection and processing methods developed for linear channels might be not optimal for nonlinear communication channels. In this Letter our focus was on proving the fact of occurrence of solitons in the OFDM signal ad study of statistics of soliton component appearances. Impact of such low energy solitons on signal dynamics and overall on transmission will be examined elsewhere.

Note, that our work is also relevant to the recently restarted studies of the so-called integrable turbulence (see e.g. [19-21] and references therein), where a random initial signal $q_{0}$ (e.g. amplified spontaneous emission) evolves in an intricate way in the plane $(z, t)$ according to (1). Statistical analysis of this evolution presented in the nonlinear spectrum can provide new insights in the complex dynamics of the optical field.

This work was supported by the Russian Science Foundation (Grant No. 17-72-30006, all authors) and, partially, by the EPSRC grant TRANSNET (SKT) and RFBR grant No. 18-02-00042 (AAG).

\section{REFERENCES}

1. G. P. Agrawal, The Nonlinear Fiber Optics (Academic press, 2007), 4th ed.

2. A. Hasegawa and Y. Kodama, Optics Lett. 15, 1443 (1990).

3. L. F. Mollenauer and J. Gordon, Solitons in optical fiber (Academic Press, 2006).

4. J. D. Ania-Castañón, T. J. Ellingham, R. Ibbotson, X. Chen, L. Zhang, and S. K. Turitsyn, Phys. Rev. Lett. 96, 023902 (2006).

5. S. K. Turitsyn, J. E. Prilepsky, S. T. Le, S. Wahls, L. L. Frumin, M. Kamalian, and S. A. Derevyanko, Optica 4, 307 (2017).

6. V. E. Zakharov and A. B. Shabat, Soviet Physics JETP 34, 62 (1972).

7. S. Manakov, Soviet Physics JETP 38, 693 (1974).

8. Y. S. Kivshar, Journal of Physics A: Mathematical and General 22, 337 (1989).

9. J. Burzlaff, Journal of Physics A: Mathematical and General 21, 561 (1988).

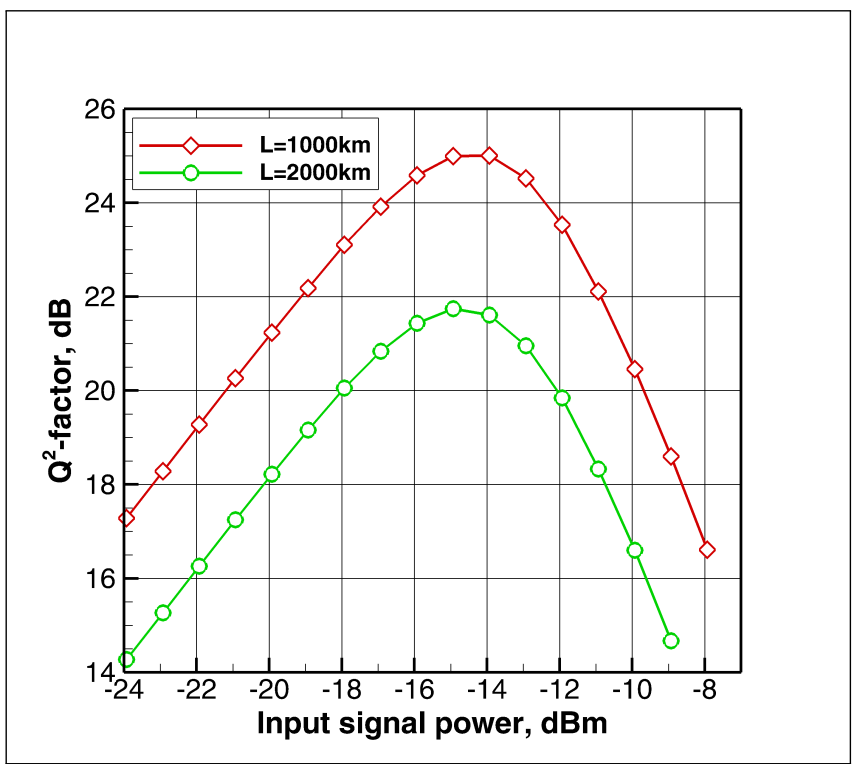

Fig. 5. The dependence of the $\mathrm{Q}^{2}$-factor on the average input power of the signal.

10. S. K. Turitsyn and S. A. Derevyanko, Physical Review A 78, 063819 (2008).

11. J. E. Prilepsky, S. A. Derevyanko, and S. K. Turitsyn, JOSA B 24, 1254 (2007).

12. S. Derevyanko and E. Small, Physical Review A 85, 053816 (2012).

13. J. G. Proakis, Digital Communications (McGraw-Hill, New York, 2000), 4th ed.

14. S. Burtsev, R. Camassa, and I. Timofeyev, Journal of computational physics 147, 166 (1998).

15. G. Boffetta and A. R. Osborne, Journal of computational physics 102, 252 (1992).

16. A. Vasylchenkova, J. E. Prilepsky, and S. K. Turitsyn, Optics Lett. 43, 3690 (2018).

17. R. Schmogrow, B. Nebendahl, M. Winter, A. Josten, D. Hillerkuss, S. Koenig, J. Meyer, M. Dreschmann, M. Huebner, C. Koos, J. Becker, W. Freude, and J. Leuthold, IEEE Photonics Technology Letters 24, 61 (2012).

18. S. Derevyanko, A. Redyuk, S. Vergeles, and S. K. Turitsyn, APL Photonics 3, 060801 (2018).

19. V. E. Zakharov, Stud. Appl. Math. 122, 219 (2009).

20. S. Randoux, P. Suret, and G. El, Scientific Reports 6, 29238 (2016).

21. P. Suret, R. El-Koussaifi, A. Tikan, C. Evain, S. Randoux, C. Szwaj, and S. Bielawski, Nature Communications 7, 13136 (2016). 


\section{FULL REFERENCES}

1. G. P. Agrawal, The Nonlinear Fiber Optics (Academic press, 2007), 4th ed.

2. A. Hasegawa and Y. Kodama, "Guiding-center soliton in optical fibers," Optics Lett. 15, 1443-1445 (1990).

3. L. F. Mollenauer and J. Gordon, Solitons in optical fiber (Academic Press, 2006).

4. J. D. Ania-Castañón, T. J. Ellingham, R. Ibbotson, X. Chen, L. Zhang, and S. K. Turitsyn, "Ultralong raman fibre lasers as virtually lossless optical media," Phys. Rev. Lett. 96, 023902 (2006).

5. S. K. Turitsyn, J. E. Prilepsky, S. T. Le, S. Wahls, L. L. Frumin, M. Kamalian, and S. A. Derevyanko, "Nonlinear fourier transform for optical data processing and transmission: advances and perspectives," Optica 4, 307-322 (2017).

6. V. E. Zakharov and A. B. Shabat, "Exact theory of two-dimensional selffocusing and one-dimensional self-modulation of waves in nonlinear media," Soviet Physics JETP 34, 62 (1972).

7. S. Manakov, "Nonlinear fraunhofer diffraction," Soviet Physics JETP $\mathbf{3 8}$, 693 (1974).

8. Y. S. Kivshar, "On the soliton generation in optical fibres," Journal of Physics A: Mathematical and General 22, 337 (1989).

9. J. Burzlaff, "The soliton number of optical soliton bound states for two special families of input pulses," Journal of Physics A: Mathematical and General 21, 561 (1988).

10. S. K. Turitsyn and S. A. Derevyanko, "Soliton-based discriminator of noncoherent optical pulses," Physical Review A 78, 063819 (2008).

11. J. E. Prilepsky, S. A. Derevyanko, and S. K. Turitsyn, "Conversion of a chirped gaussian pulse to a soliton or a bound multisoliton state in quasi-lossless and lossy optical fiber spans," JOSA B 24, 1254-1261 (2007).

12. S. Derevyanko and E. Small, "Nonlinear propagation of an optical speckle field," Physical Review A 85, 053816 (2012).

13. J. G. Proakis, Digital Communications (McGraw-Hill, New York, 2000), 4th ed.

14. S. Burtsev, R. Camassa, and I. Timofeyev, "Numerical algorithms for the direct spectral transform with applications to nonlinear schrödinger type systems," Journal of computational physics 147, 166-186 (1998).

15. G. Boffetta and A. R. Osborne, "Computation of the direct scattering transform for the nonlinear schroedinger equation," Journal of computational physics 102, 252-264 (1992).

16. A. Vasylchenkova, J. E. Prilepsky, and S. K. Turitsyn, "Contour integrals for numerical computation of discrete eigenvalues in the zakharov-shabat problem," Optics Lett. 43, 3690-3693 (2018).

17. R. Schmogrow, B. Nebendahl, M. Winter, A. Josten, D. Hillerkuss, S. Koenig, J. Meyer, M. Dreschmann, M. Huebner, C. Koos, J. Becker, W. Freude, and J. Leuthold, "Error vector magnitude as a performance measure for advanced modulation formats," IEEE Photonics Technology Letters 24, 61-63 (2012).

18. S. Derevyanko, A. Redyuk, S. Vergeles, and S. K. Turitsyn, "Visualisation of extreme value events in optical communications," APL Photonics 3, 060801 (2018).

19. V. E. Zakharov, "Turbulence in integrable systems," Stud. Appl. Math. 122, 219-234 (2009).

20. S. Randoux, P. Suret, and G. El, "Inverse scattering transform analysis of rogue waves using local periodization procedure," Scientific Reports 6, 29238 (2016).

21. P. Suret, R. El-Koussaifi, A. Tikan, C. Evain, S. Randoux, C. Szwaj, and S. Bielawski, "Single-shot observation of optical rogue waves in integrable turbulence using time microscopy," Nature Communications 7, 13136 (2016). 